\title{
Maximal Elements and Coincident Points for Couple-Majorized Mappings in Product Topological Vector Spaces
}

\author{
P. Deguire and Xian-Zhi Yuan
}

\begin{abstract}
By introducing a concept called 'couple-majorized mapping' in product topological vector spaces we establish existence theorems of maximal elements for couple-majorized setvalued families with arbitrary index set. As applications, some component coincidence and fixed points theorems are given which could be regarded as product extension version of the Fan-Browder fixed point theorem and related coincidence theorems. Finally, a coincidence theorem is used to derive the Sion minimax inequality in the case of two functions.

Keywords: Couple-majorized mappings, Fan-Browder fixed point theorem, maximal elements, coincidence points, minimax inequalities, Knaster-Kuratowski-Mazurkiewicz lemma and product spaces
\end{abstract}

AMS subject classiflcation: 47 H 04, 49 J 35, 90 D 06

\section{Introduction}

It is well-known that each fixed point theorem has an equivalent version as existence theorem of maximal elements. We recall that a point $x \in X$ is a maximal element of a set-valued mapping $F$ from a topological space $X$ into another topological space $Y$ if $F(x)=\emptyset$. The existence of maximal elements for mappings in topological (vector) spaces and their important applications to mathematical economics have been studied by many authors in both mathematics and economics (see, for example, Aubin [1], Ben-El-Mechaiekh and Deguire [2], Deguire and Lassonde [5], Deguire et al. [6], Ding et al [7], Hildenbrand and Sonnenschein [12], Lassonde and Schenkel [15], Mas-Colell [16 - 17], Tan and Yuan [21], Tarafdar [22], Toussaint [24], Tulcea [25], and Yannelis and Prabhakar [26]). Recently, by the existence of selection functions for set-valued mappings with open fibers in product spaces, Deguire and Lassonde [5] have given some fixed point theorems in product spaces for both compact and non-compact domains. Moreover, by introducing so-called $L_{S}$-majorized mappings in product spaces, some existence theorems of maximal elements have been established by Deguire et al [6].

However all existence theorems of maximal elements mentioned above only concern one family of set-valued mappings in product spaces. Furthermore, it is clear that each

P. Deguire: Univ. Moncton, Dep. Math. Stat., Moncton, New Brunswick, Canada E1A 3E9. Xian-Zhi Yuan: Univ. Queensland, Dep. Math., Brisbane, Australia 4072.

This work was done while the second author was visiting The University of Moncton. 
coincidence theorem in product spaces has an equivalent version of maximal elements in product spaces which corresponds to the existence of maximal elements for two setvalued families:

This observation leads us to study in this paper coincidence theorems from the point of view of existence theorems of maximal elements in product spaces instead of using continuous selection theorems for set-valued mappings with convex (maybe empty) values and open fibers posed by Deguire and Lassonde [5]. Our method in this paper enables us to study the existence of coincidence theorems for two families of large classes of set-valued mappings as an immediate application of existence theorems of maximal elements for two families of set-valued mappings. Precisely, by introducing a concept called couple-majorized mapping for two families in product spaces, we first establish existence theorems of maximal elements for couple-majorized families in Section 2. As applications, some coincidence and fixed point theorems are then given in Section 3 . In particular, our fixed point theorem could be regarded as component version of the FanBrowder fixed point theorem in product spaces. Finally, the Sion minimax inequality for two functions is derived as an application of our coincidence theorem. These results unify and improve most of the corresponding results in the literature and our approach employed in this paper is different from those used or given by Ben-El-Mechaiekh and Deguire [2], Deguire and Lassonde [5], Ding et al [7], Hildenbrand and Sonnenschein [12], Lassonde and Schenkel [15], Mas-Colell [16 - 17], Tan and Yuan [21], Tarafdar [22], Toussaint [24], Tulcea [25], and Yannelis and Prabhakar [26].

We now introduce some notation and definitions.

If $X$ is a set, then $2^{X}$ denotes the family of all subsets of $X$. Throughout this paper, the term ' $m a p$ ' always means a single-valued one. If $X=\prod_{i \in I} X_{i}$ is the product space of a family of spaces $X_{i} \quad(i \in I)$, then for each element $x \in X$ we shall denote it as $x=\left\{x_{i}\right\}_{i \in I}$, where $x_{i}$ means the $i$ th component under the project operation from $X$ into $X_{i}$, for each $i \in I$.

Let $X$ be a topological space and $Y$ a non-empty set in a topological vector space. Then a mapping $A: X \rightarrow 2^{Y}$ is said to have

- convex values if $A(x)$ is convex in $Y$ for all $x \in X$

- open fibers if $A^{-1}(y)=\{x \in X: y \in A(x)\}$ is open in $X$ for each $y \in Y$.

If $U$ is a non-empty subset of $X$, then int $X U$ denotes the interior of $U$ in $X$. We shall denote it for convenience simply by int $U$ if no confusion can be. Also all topological spaces are assumed to be Hausdorff unless otherwise specified.

Now we have the following

Deflnition 1.1. Let $X=\Pi_{j \in J} X_{j}$ and $Y=\Pi_{i \in I} Y_{i}$ be two product spaces of nonempty convex subsets $X_{j}$ and $Y_{i}$ in topological vector spaces $E_{j}$ and $F_{i}$, respectively, where $I$ and $J$ are any given index sets. For each $i \in I$ and $j \in J$, let $A_{i}: X \rightarrow 2^{Y_{i}}$ and $B_{j}: Y \rightarrow 2^{X_{j}}$ be two mappings. Then the families $\left\{A_{i}\right\}_{i \in I}$ and $\left\{B_{j}\right\}_{j \in J}$ are said to be couple-majorized if for any given index pair $(i, j) \in I \times J$ and for any element pair $(x, y) \in X \times Y$ with $A_{i}(x) \neq \emptyset$ and $B,(y) \neq \emptyset$ there exist mappings $A_{i(x)}: X \rightarrow 2^{Y_{i}}$ and $B_{j(y)}: Y \rightarrow 2^{X_{j}}$ and non-empty open neighbourhoods $N(x)$ of $x$ in $X$ and $N(y)$ of $y$ in $Y$ such that the following conditions are satisfied: 
(i) Both $A_{i(x)}$ and $B_{j(y)}$ have (maybe empty) convex values and open fibers.

(ii) $A_{i}(z) \subset A_{i(x)}(z)$ and $B_{j}(w) \subset B_{j(y)}(w)$ for all $(z, w) \in N(x) \times N(y)$.

(iii) $\left(u_{j}, v_{i}\right) \notin\left(B_{j(y)}(v), A_{i(x)}(u)\right)$ for all $(u, v) \in X \times Y$.

Also we shall call the mapping pair $\left(A_{i(x)}, B_{j(y)}\right)$ which satisfies these conditions a couple-majorant of the mapping pair $\left(A_{i}, B_{j}\right)$ at the point $(x, y) \in X \times Y$ throughout this paper.

\section{Maximal elements for couple-majorized families}

Let $E$ be a topological vector space. Then a polytope of $E$ is the convex hull of any nonempty finite subset of $E$. By the partition of unity for paracompact spaces, the following result can be easily proved (see, for example, Dugundji and Granas [8], Yannelis and Prabhakar [26], and Deguire and Lassonde [5] for more recent results in this direction).

Lemma 2.1. Let $X$ be a paracompact topological space and $Y$ a non-empty convex subset of a topological vector space $E$. Suppose $F: X \rightarrow 2^{Y}$ is a set-valued mapping with non-empty convex values and open fibers. Then there exists a continuous map $f: X \rightarrow Y$ such that $f(x) \in F(x)$ for each $x \in X$. In particular, if $X$ is compact, there exists a polytope $D$ in $Y$ such that $f(X):=\cup_{x \in X} f(x) \subset D$.

By Lemma 2.1 and the classical Brouwer fixed point theorem, it was Browder, who first gave the following form of a fixed point theorem.

Lemma 2.2 (Fan-Browder fixed point theorem, see [3]). Let $X$ be a non-empty compact and convex subset of a topological vector space $E$ and $F: X \rightarrow 2^{X}$ a set-valued mapping with non-empty convex values and open fibers. Then $F$ has a fixed point.

But really, seven years before the paper [3] of Browder, it was Fan, who as first person proved in [9] an equivalent form of the Browder fixed point theorem by using his extension to infinite dimensional topological vector spaces of the classical KnasterKuratowski-Mazurkiewicz lemma (in short, KKM lemma) given in [13]. Since then, this result is often called Fan-Browder (or, Browder-Fan) fixed point theorem.

As applications of Lemmas 2.1 and 2.2, we have the following existence theorem of maximal elements for a pair of mappings which may not have convex values nor open fibers.

Theorem 2.3. Let $X$ and $Y$ be non-empty compact convex subsets of topological vector spaces $E$ and $F$, respectively. Suppose $A: X \rightarrow 2^{Y}$ and $B: Y \rightarrow 2^{X}$ to be couple-majorized mappings. Then there exists $x_{0} \in X$ with $A\left(x_{0}\right)=\emptyset$ or $y_{0} \in Y$ with $B\left(y_{0}\right)=\emptyset$.

Proof. Suppose contrary that, for each $x \in X$ and $y \in Y, A(x) \neq \emptyset$ and $B(y) \neq$ 0 . Then there exist a non-empty open neighbourhood $N(x)$ of $x$ in $X$ and an open neighbourhood $N(y)$ of $y$ in $Y$, and two mappings $A_{x}: X \rightarrow 2^{Y}$ and $B_{y}: Y \rightarrow 2^{Y}$ such that

(a) $A(z) \subset A_{x}(z)$ for each $z \in N(x)$ and $B(z) \subset B_{y}(z)$ for each $z \in N(y)$ 
(b) $(u, v) \notin\left(B_{y}(v), A_{x}(u)\right)$ for each $(u, v) \in X \times Y$

(c) $A_{x}^{-1}(u)$ and $B_{y}^{-1}(v)$ are open in $X$ and $Y$, respectively, for each $u \in Y$ and $v \in X$.

Since $X$ and $Y$ are regular, for each $x \in X$ and $y \in Y$ let $O(x)$ and $O(y)$ be open neighbourhoods of $x$ in $X$ and $y \in Y$ such that $\overline{O(x)} \subset N(x)$ and $\overline{O(y)} \subset N(y)$. Note that since $X$ is compact and $X=\cup_{x \in X} O(x)$, there exist elements $x_{1}, \ldots, x_{n} \in X$ such that $X=\cup_{i=1}^{n} O\left(x_{i}\right)$. Define mappings $A_{i}: X \rightarrow 2^{Y} \quad(i=1,2, \ldots, n)$ by

$$
A_{i}(x)=\left\{\begin{array}{ll}
A_{x_{i}}(x) & \text { if } x \in \overline{O\left(x_{i}\right)} \\
Y & \text { if } x \in X \backslash \overline{O\left(x_{i}\right)} .
\end{array} \quad(x \in X)\right.
$$

Then we have:

(a) ${ }^{\prime} A_{i}$ is convex-valued

(b) $A(x) \subset A_{i}(x)$ for each $x \in X$

(c) $A_{i}^{-1}(u)$ is open in $X$, for each $u \in Y$, as .

$$
\begin{aligned}
A_{i}^{-1}(u) & =\left\{x \in \overline{O\left(x_{i}\right)}: u \in A_{x_{i}}(x)\right\} \cup\left(X \backslash \overline{O\left(x_{i}\right)}\right) \\
& =\left(A_{x_{i}}^{-1}(u) \cap \overline{O\left(x_{i}\right)}\right) \cup\left(X \backslash \overline{O\left(x_{i}\right)}\right) \\
& =\left(A_{x_{i}}^{-1}(u) \cap \overline{O\left(x_{i}\right)}\right) \cap\left(\overline{O\left(x_{i}\right)} \cup\left(X \backslash \overline{O\left(x_{i}\right)}\right)\right. \\
& =A_{x_{i}}^{-1}(u) \cup\left(X \backslash \overline{O\left(x_{i}\right)}\right)
\end{aligned}
$$

which is open by property (c) above. Now define $A^{\prime}: X \rightarrow 2^{Y}$ by $A^{\prime}(x)=\cap_{i=1}^{n} A_{i}(x)$ for each $x \in X$. Clearly, we have:

(a)" $A^{\prime}$ is (maybe empty) convex-valued

(b)" $A(x) \subset A^{\prime}(x)$ for each $x \in X$

$(\text { c) })^{\prime \prime}\left(A^{\prime}\right)^{-1}(y)$ is open in $X$, for each $y \in Y$, as

$$
\left(A^{\prime}\right)^{-1}(y)=\left\{x \in X: y \in \cap_{i=1}^{n} A_{i}(x)\right\}=\cap_{i=1}^{n} A_{i}^{-1}(y) .
$$

Note that since $Y$ is also compact and $Y=\cup_{y \in Y} O(y)$, there exist elements $y_{1}, \ldots, y_{m} \in$ $Y$ such that $Y=\cup_{i=1}^{m} O\left(y_{i}\right)$. Define mappings $B_{i}: Y \rightarrow 2^{X} \quad(i=1,2, \ldots, m)$ by

$$
B_{i}(y)=\left\{\begin{array}{ll}
B_{y_{i}}(y) & \text { if } y \in \overline{O\left(y_{i}\right)} \\
X & \text { if } y \in Y \backslash \overline{O\left(y_{i}\right)}
\end{array} \quad(y \in Y)\right.
$$

Now, similarly to the arguments used for the mapping $A^{\prime}$, we also have:

(i)' $B_{i}$ is (maybe empty) convex-valued

(ii) $B(y) \subset B_{i}(y)$ for each $y \in Y$

(iii) $B_{i}^{-1}(x)$ is open in $Y$, for each $x \in X$. 
Now we define a mapping $B^{\prime}: Y \rightarrow 2^{X}$ by $B^{\prime}(x)=\cap_{i=1}^{m} B_{i}(y)$, for each $y^{\prime} \in Y$. Then we have:

(i)" $B^{\prime}$ is convex-valued

(ii) $B(y) \subset B^{\prime}(y)$ for each $y \in Y$

(iii)" $\left(B^{\prime}\right)^{-1}(x)$ is open in $Y$, for each $x \in X$, as

$$
\left(B^{\prime}\right)^{-1}(x)=\left\{y \in Y: x \in \cap_{i=1}^{n} B_{i}(y)\right\}=\cap_{i=1}^{n} B_{i}^{-1}(x) .
$$

Moreover, we also have:

(d) for each $x \in X$ and $y \in Y, x \notin B^{\prime}(y)$ or $y \notin A^{\prime}(x)$ by the definition of $A^{\prime}$ and $B^{\prime}$

(e) both mappings $A^{\prime}$ and $B^{\prime}$ have non-empty values as $\emptyset \neq A(x) \subset A^{\prime}(x)$ and

$\emptyset \neq B(y) \subset B^{\prime}(y)$ for each $x \in X$ and $y \in Y$.

As $X$ is compact and $A^{\prime}$ has non-empty convex values and open fibers, Lemma 2.1 implies that there exist a polytope $D$ in $Y$ and a continuous (single-valued) function $f: X \rightarrow D$ such that $f(x) \in A^{\prime}(x)$ for each $x \in X$. Now the mapping $F: X \rightarrow 2^{X}$ defined by $F(x)=B^{\prime}(f(x))$ for each $x \in X$ has the following properties:

- $F(x)$ is non-empty convex for each $x \in X$

- $F^{-1}(y):=\left\{x \in X: y \in\left(B^{\prime}\right)^{-1}(f(x))\right\}=f^{-1}\left(\left(B^{\prime}\right)^{-1}(y)\right)$ is open in $X$ for each $y \in X$ by property (iii)".

Thus Lemma 2.2 implies that there exits $x_{0} \in X$ such that $x_{0} \in F\left(x_{0}\right)=B^{\prime}\left(f\left(x_{0}\right)\right)$. Let $y_{0}=f\left(x_{0}\right)$. Then $x_{0} \in B^{\prime}\left(y_{0}\right)$ and $y_{0} \in A^{\prime}\left(x_{0}\right)$ as $f\left(x_{0}\right) \in A^{\prime}\left(x_{0}\right)$, but this contradicts (d). Therefore the conclusion follows and we completed the proof

Let $X=Y$ in Theorem 2.3. As a direct application of the Fan-Browder fixed point theorem, we have the following existence of maximal elements.

Theorem 2.4. Let $X$ be a non-empty compact convex subset of a topological vector space $E$ and $A: X \rightarrow 2^{X}$ a set-valued mapping. Suppose that for each $x \in X$ with $A(x) \neq \emptyset$ there exist a non-empty open neighbourhood $N(x)$ of $x$ in $X$ and a set-valued mapping $A_{x}: X \rightarrow 2^{X}$ which has (maybe empty) convex values such that

(i) $A(z) \subset A_{x}(z)$ for all $z \in N(x)$

(ii) $A_{x}^{-1}(y)=\left\{z \in X: y \in A_{x}(z)\right\}$ is open for each $y \in X$

(iii) $z \notin A_{x}(z)$ for all $z \in X$.

Then there exists $x_{0} \in X$ such that $A\left(x_{0}\right)=\emptyset$.

Proof. By following the same idea used in the proof of Theorem 2.3, we will prove the conclusion. Suppose contrary that, for each $x \in X, A(x) \neq \emptyset$. Then for each $x \in X$ there exist a non-empty open neighbourhood $N(x)$ of $x$ in $X$ and a mapping $A_{x}: X \rightarrow 2^{X}$ which satisfy conditions (i) - (iii) of Theorem 2.4 .

Note that $X$ is compact. For each $x \in X$, let $O(x)$ be an open neighbourhood of $x$ in $X$ such that $\overline{O(x)} \subset N(x)$. Then $X=\cup_{x \in X} O(x)$. By the compactness of 
$X$, there exist elements $x_{1}, \ldots, x_{n} \in X$ such that $X=\cup_{i=1}^{n} O\left(x_{i}\right)$. Define mappings $A_{i}: X \rightarrow 2^{X} \quad(i=1,2, \ldots, n)$ by

$$
A_{i}(x)=\left\{\begin{array}{ll}
A_{x_{i}}(x) & \text { if } x \in \overline{O\left(x_{i}\right)} \\
X & \text { if } x \in X \backslash \overline{O\left(x_{i}\right)}
\end{array} \quad(x \in X)\right.
$$

Then by similar arguments used in the proof of Theorem 2.3 we have:

(a) ${ }^{\prime} A_{i}$ is non-empty convex-valued

(b) $A(x) \subset A_{i}(x)$ for each $x \in X$

(c) $A_{i}^{-1}(y)$ is open in $X$, for each $y \in X$.

Now define a mapping $A^{\prime}: X \rightarrow 2^{X}$ by $A^{\prime}(x)=\cap_{i=1}^{n} A_{i}(x)$ for each $x \in X$. Clearly we also have:

(a) ${ }^{\prime \prime} A^{\prime}$ is non-empty convex-valued

(b)" $A(x) \subset A^{\prime}(x)$ for each $x \in X$

$(c)^{\prime \prime}\left(A^{\prime}\right)^{-1}(y)$ is open in $X$, for each $y \in X$, as

$$
\left(A^{\prime}\right)^{-1}(y)=\left\{x \in X: y \in \cap_{i=1}^{n} A_{i}(x)\right\}=\cap_{i=1}^{n} A_{i}^{-1}(y) .
$$

As $X$ is compact and $A^{\prime}$ has non-empty convex values and open fibers, Lemma 2.2 implies that $A^{\prime}$ has a fixed point. On the other hand, it is clear that $x \notin A^{\prime}(x)$ for all $x \in X$ by its definition. This contradiction shows that there must exist $x_{0} \in X$ such that $A\left(x_{0}\right)=0$

As an application of Theorem 2.3, we have the following existence theorem of maximal elements for two families which are coupled-majorized mappings in product spaces.

Theorem 2.5. Let $X=\Pi_{j \in J} X_{j}$ and $Y=\Pi_{i \in I} Y_{i}$ product spaces of non-empty compact convex subsets $X_{j}$ and $Y_{i}$ of topological vector spaces $E_{j}$ and $F_{i}$, respectively, $I$ and $J$ being arbitrary index sets. Suppose $A_{i}: X \rightarrow 2^{Y_{i}}$ and $B_{j}: Y \rightarrow 2^{X_{i}}$ are couple-majorized families of mappings such that

$$
\begin{aligned}
& \bigcup_{j \in J}\left\{y \in Y: B_{j}(y) \neq \emptyset\right\}=\bigcup_{j \in J} \operatorname{int}\left\{y \in Y: B_{j}(y) \neq \emptyset\right\} \\
& \bigcup_{i \in I}\left\{x \in X: A_{i}(x) \neq \emptyset\right\}=\bigcup_{i \in I} \operatorname{int}\left\{x \in X: A_{i}(x) \neq \emptyset\right\}
\end{aligned}
$$

Then there exists at least one $x \in X$ such that $A_{i}(x)=\emptyset$ for all $i \in I$ or one $y \in Y$ such that $B_{j}(y)=\emptyset$ for all $j \in J$.

Proof. For each $i \in I$, define a mapping $A_{i}^{\prime}: X \rightarrow 2^{Y}$ by

$$
A_{i}^{\prime}(x)=\prod_{j \in I, j \neq i} Y_{j} \times A_{i}(x) \quad(x \in X)
$$


and for each $x \in X$ define the index subset $I(x)=\left\{i \in I: A_{i}(x) \neq \emptyset\right\}$. Now we define the mapping $A: X \rightarrow 2^{Y}$ by

$$
A(x)= \begin{cases}\cap_{i \in I(x)} A_{i}^{\prime}(x) & \text { if } I(x) \neq \emptyset \\ \emptyset & \text { if } I(x)=\emptyset\end{cases}
$$

Similarly, for each $j \in J$ define the mapping $B_{j}^{\prime}: Y \rightarrow 2^{X_{j}}$ by

$$
B_{i}^{\prime}(y)=\prod_{i \in J, i \neq j} X_{j} \times B_{i}(y) \quad(y \in Y)
$$

and for each $y \in Y$ define the index subset $J(y)=\{j \in J: B,(y) \neq \emptyset\}$. Now we define the mapping $B: Y \rightarrow 2^{X}$ by

$$
B(y)= \begin{cases}\bigcap_{j \in J(y)} B_{i}^{\prime}(y) & \text { if } J(y) \neq \emptyset \\ \emptyset & \text { if } J(y)=\emptyset\end{cases}
$$

Then we shall show that the pair $(A, B)$ is couple-majorized. Let $(x, y) \in X \times Y$. We may assume that $I(x) \neq \emptyset$ and $J(y) \neq \emptyset$, otherwise the conclusion follows. Then

$$
x \in \bigcup_{i \in I}\left\{x \in X: A_{i}(x) \neq \emptyset\right\} \quad \text { and } \quad y \in \bigcup_{j \in J}\{y \in Y: B ;(y) \neq \emptyset\} .
$$

Since $\left(A_{i}, B_{j}\right)$ is couple-majorized, there exist open neighbourhoods $N(x)$ of $x$ in $X$ and $N(y)$ of $y$ in $Y$, and a pair of a couple-majorant mappings $C_{i}: X \rightarrow 2^{Y_{i}}$ and $D_{j}: Y \rightarrow 2^{X_{j}}$ at $(x, y)$ such that we have:

(a) $A_{i}(z) \subset C_{i}(z)$ for each $z \in N(x)$ and $B_{j}(z) \subset D_{j}(z)$ for each $z \in N(y)$

(b) $\left(u_{j}, v_{i}\right) \notin\left(D_{j}(v), C_{i}(u)\right)$ for each $(u, v) \in X \times Y$

(c) $C_{i}^{-1}\left(v_{i}\right)$ and $D_{j}^{-1}\left(u_{j}\right)$ are open in $X$ and $Y$, respectively, for each $v_{i} \in Y_{i}$ and $u_{j} \in X_{j}$.

By our assumptions, without loss of generality, we may assume that

$$
N(x) \subset \operatorname{int}\left\{x \in X: A_{i}(x) \neq \emptyset\right\} \quad \text { and } \quad N(y) \subset \operatorname{int}\left\{y \in Y: B_{j}(y) \neq \emptyset\right\} .
$$

Thus $A_{i}(z) \neq \emptyset$ for each $z \in N(x)$ and $B_{j}(z) \neq \emptyset$ for each $z \in N(y)$. Now we define mappings $C_{x}: X \rightarrow 2^{Y}$ and $D_{y}: Y \rightarrow 2^{X}$ by

$$
C_{x}(z)=\prod_{j \in I, j \neq i} Y_{j} \times C_{i}(z) \quad \text { and } \quad D_{y}(z)=\prod_{i \in J, i \neq j} X_{j} \times D_{j}(z)
$$

for each $z$ in $X$ and $Y$, respectively. Then we claim that $\left(C_{x}, D_{y}\right)$ is a couple-majorant of the pair $(A, B)$ at $(x, y) \in X \times Y$. Indeed, we have:

(i) $A(z)=\cap_{k \in I(z)} A_{k}^{\prime}(z) \subset A_{i}^{\prime}(z) \subset C_{x}(z)$ for each $z \in N(x)$ 
(ii) $B(z)=\cap_{k \in J(z)} B_{k}^{\prime}(z) \subset B_{i}^{\prime}(z) \subset D_{y}(z)$ for each $z \in N(y)$

(iii) $C_{x}^{-1}(v)=\left(C_{i}^{\prime}\right)^{-1}(v)$ and $D_{y}^{-1}(u)=\left(D_{j}^{\prime}\right)^{-1}(u)$ are open in $X$ and $Y$, respectively, for each $u \in X$ and $v \in Y$

(iv) $(u, v) \notin D_{y}(v) \times C_{x}(u)$ for each $u \in X$ and $v \in Y$.

Therefore $\left(C_{x}, D_{y}\right)$ is a couple-majorant of $(A, B)$ at $(x, y)$. Thus $(A, B)$ satisfies all hypotheses of Theorem 2.3 and there exists at least one $x \in X$ with $A(x)=\emptyset$ or one $y \in Y$ with $B(y)=\emptyset$, i.e. $A_{i}(x)=\emptyset$ for all $i \in I$ or $B_{j}(y)=\emptyset$ for all $j \in J$ and the proof is completed

As an application of Theorem 2.4, we also have the following result.

Theorem 2.6. Let $X=\Pi_{i \in I} X_{i}$ be the product space of a family of non-empty compact convex subsets $X_{i}$ of topological vector spaces $E_{i}$, I being an arbitrary index set. Let $\left\{A_{i}\right\}_{i \in I}$ be a family of set-valued mappings $A_{i}: X \rightarrow 2^{X_{i}}$ such that

$$
\bigcup_{i \in I}\left\{x \in X: A_{i}(x) \neq \emptyset\right\}=\bigcup_{i \in I} \operatorname{int}\left\{x \in X: A_{i}(x) \neq \emptyset\right\}
$$

Suppose that for each $i \in I$ and $\dot{x} \in X$ with $A_{i}(x) \neq \emptyset$ there exist a non-empty open neighbourhood $N(x)$ of $x$ in $X$ and a set-valued mapping $A_{i, x}: X \rightarrow 2^{X_{i}}$ which takes (maybe empty) convex values such that

(i) $A_{i}(z) \cdot \subset A_{i, x}(z)$ for all $z \in N(x)$

(ii) $z_{i} \notin A_{i, x}(z)$ for all $z . \in X$

(iii) $A_{i, x}^{-1}(y)=\left\{z \in X: y \in A_{i, x}(z)\right\}$ is open in $X$, for each $y \in X_{i}$.

Then there exists at least one $\hat{x} \in X$ such that $A_{i}(\hat{x})=\emptyset$ for all $i \in I$.

Proof. By following similar arguments used in the proof of Theorem 2.5, for each $i \in I$ we define a mapping $A_{i}^{\prime}: X \rightarrow 2^{X}$ by

$$
A_{\mathrm{i}}^{\prime}(x)=\Pi_{j \in I, j \neq i} X_{j} \times A_{\mathrm{i}}(x) \quad(x \in X)
$$

and for each $x \in X$ we define a index subset $I(x)=\left\{i \in I: A_{i}(x) \neq \emptyset\right\}$. Now define a mapping $A: X \rightarrow 2^{X}$ by

$$
A(x)= \begin{cases}\cap_{i \in I(x)} A_{i}^{\prime}(x) & \text { if } I(x) \neq \emptyset \\ \emptyset & \text { if } I(x)=\emptyset .\end{cases}
$$

Then we shall show that $A$ satisfies all conditions of Theorem 2.4. Without loss of generality, we may assume that $I(x) \neq \emptyset$ (otherwise the conclusion follows). Then $x \in \cup_{i \in I}\left\{x \in X: A_{i}(x) \neq \emptyset\right\}$ and there exists $i \in I$ such that $A_{i}(x) \neq \emptyset$. Thus there exist an open neighbourhood $N(x)$ of $x$ in $X$ and a set-valued mapping $A_{i, x}: X \rightarrow 2^{X_{i}}$ which satisfies all conditions (i) - (iii) of Theorem 2.6. By our assumptions, we can also assume that $N(x) \subset \operatorname{int}\left\{x \in X: A_{i}(x) \neq \emptyset\right\}$. Thus $A_{i}(z) \neq \emptyset$ for each $z \in N(x)$. Now define a mapping $A_{x}: X \rightarrow 2^{X}$ by

$$
A_{x}(z)=\Pi_{j \in I, j \neq i} X_{j} \times A_{i, x}(z) \quad(z \in X) .
$$


Then it is clear that we have:

(i) $A(z)=\cap_{k \in I(x)} A_{k}^{\prime}(z) \subset A_{i}^{\prime}(z) \subset A_{x}(z)$ for each $z \in N(x)$, and $A_{x}$ takes (maybe empty) convex values

(ii) $A_{x}^{-1}(v)=\left(A_{i, x}^{\prime}\right)^{-1}(v)$ which is open in $X$ for each $v \in X$

(iii) $z \notin A_{x}(z)$ for all $z \in X$.

Therefore we have shown that all hypotheses of Theorem 2.4 are satisfied and there exists an $\hat{x} \in X$ such that $A(\hat{x})=\emptyset$. Thus the proof is completed

Remark 2.7. Let $X$ and $Y$ be two non-empty sets; $A: X \rightarrow 2^{Y}$ a set-valued mapping and $S: Y \rightarrow X$ a single-valued map. We can definite their composition $A \circ S$ as a map from $Y$ into $2^{Y}$ by $(A \circ S)(y)=A(S(y))$ for each $y \in Y$. When $X$ is a compact topological space instead of having a linear structure and the mappings $B_{j}$ in Theorems 2.3 and 2.5 are replaced by a continuous single-valued mapping $S$, some existence theorems of maximal elements for $L_{S}$-majorized mappings in product spaces have been established recently by authors' joint research work [6]. Note that both concepts 'couple-majorized' and ' $L_{S}$-majorized' are independent to each other, thus existence theorems of maximal elements in this paper are independent of those results given in $[6]$.

\section{Coincidence and fixed point theorems in product spaces}

A coincidence theorem is a theorem asserting that if mappings $S: X \rightarrow 2^{\gamma}$ and $T: Y \rightarrow 2^{X}$ have non-empty values and satisfy certain other conditions, then there exist $x_{0} \in X$ and $y_{0} \in Y$ such that $y_{0} \in S x_{0}$ and $x_{0} \in T y_{0}$.

In this section, as applications of existence theorems for maximal elements in Section 2 , we shall derive some coincidence and fixed point theorems in product spaces. For convenience, we first recall the following definition which has been used by Deguire in [4] (see also [5, 6]).

Definition 3.1. Let $X$ be a topological space and $I$ any index set. For each $i \in I$, suppose that $Y_{i}$ is a non-empty convex subset of a topological vector space $F_{i}$ and $A_{i}: X \rightarrow 2^{Y_{i}}$ a set-valued mapping. Then $\left\{A_{i}\right\}_{i \in I}$ is said to be a $K F$ family if the following assumptions are satisfied:

(i) For each $i \in I, A_{i}(x)$ is convex for each $x \in X$.

(ii) For each $x \in X$, there exists $i \in I$ such that $A_{i}(x) \neq \emptyset$.

(iii) For each $i \in I, A_{i}^{-1}\left(y_{i}\right)$ is open in $X$ for each $y_{i} \in Y_{i}$.

Now we have the following coincidence theorem in product spaces.

Theorem 3.1. Let $X=\prod_{j \in J} X_{j}$ and $Y=\prod_{i \in I} Y_{i}$ be product spaces of non-empty compact convex subsets $X_{j}$ and $Y_{i}$ in topological vector spaces $E_{j}$ and $F_{i}$, respectively, $I$ and $J$ being arbitrary index sets. Suppose that both $\left\{A_{i}\right\}_{i \in I}$ and $\left\{B_{j}\right\}_{j \in J}$ are $K F$ families. Then there exist an index pair $\left(i_{0}, j_{0}\right) \in I \times J$ and an element pair $(\hat{x}, \hat{y}) \in$ $X \times Y$ such that $\hat{y}_{i_{0}} \in A_{i_{0}}(\hat{x})$ and $\hat{x}_{j_{0}} \in B_{j_{0}}(\hat{y})$. 
Proof. Suppose the conclusion is not true. Then $\left\{A_{i}\right\}_{i \in I}$ and $\left\{B_{j}\right\}_{j \in J}$ are couplemajorized. By Theorem 2.5, there exist either an $\hat{x} \in X$ with $A_{i}(\hat{x})=\emptyset$ for all $i \in I$ or an $\hat{y} \in Y$ with $B_{j}(\hat{y})=\emptyset$ for all $j \in J$, which contradicts that both $\left\{A_{i}\right\}_{i \in I}$ and $\left\{B_{j}\right\}_{j \in J}$ are KF families. Thus the conclusion follows

We wish to note that the conclusion of Theorem 3.1 was also given by Deguire and Lassonde [5], but in a different way which mainly depends on the continuous selection theorem of $\mathrm{KF}$ families. However, our approach used here is different from theirs as Theorem 3.1 is a consequence of our Theorem 2.5 which gives the existence of maximal elements for couple-majorized mappings.

As an application of Theorem 2.6, we have the following component fixed point theorem which could be regarded as an extension of the Fan-Browder fixed point theorem in product spaces.

Theorem 3.2. Let $X=\prod_{i \in I} X_{i}$ be the product space of non-empty compact convex sets $X_{i}$ in a topological vector space $E_{i}$, $I$ being an arbitrary index set. Suppose that $\left\{A_{i}: X \rightarrow 2^{X_{i}}\right\}_{i \in I}$ is a $K F$ family. Then there exist an index $i_{0} \in I$ and an element $\hat{x} \in X$ such that $\hat{x}_{i_{0}} \in A_{i_{0}}(\hat{x})$.

Proof. Suppose the conclusion were not true. Then, by Theorem 2.6, there exists an element $\hat{x} \in X$ with $A_{i}(\hat{x})=\emptyset$ for all $i \in I$. Of course, this contradicts with that $\left\{A_{i}\right\}_{i \in I}$ is a KF family. Thus there must exist an index $i \in I$ and an element $\hat{x} \in X$ with $\hat{x}_{i_{0}} \in A_{i_{0}}(\hat{x})$ and the proof is completed

An an immediate consequence of Theorem 3.2, we shall the derive Sion minimax inequality in the case of two functions in [20].

Corollary 3.3 (Sion minimax inequality). Let $X$ and $Y$ be non-empty compact convex subsets of topological vector spaces $E$ and $F$, respectively. Suppose functions $f, g: X \times Y \rightarrow \mathbb{R} \cup\{-\infty,+\infty\}$ satisfy the following conditions:

(i) $f(x, y) \leq g(x, y)$ for each $(x, y) \in X \times Y$.

(ii) For each $x \in X, f(x, \cdot)$ is quasi-concave, and for each $y \in Y, f(\cdot, y)$ is lower semicontinuous.

(iii) For each fixed $x \in X, g(x, \cdot)$ is upper semicontinuous, and for each fixed $y \in Y$, $g(\cdot, y)$ is quasi-convex.

Then we have

$$
\min _{x \in X} \sup _{y \in Y} f(x, y) \leq \sup _{y \in Y} \min _{x \in X} g(x, y) .
$$

Proof. Set $\lambda_{0}=\min _{x \in X} \sup _{y \in Y} f(x, y)$ and, for each given $\varepsilon>0, \lambda_{\varepsilon}=\lambda_{0}-\varepsilon$. Define mappings $A: X \rightarrow 2^{Y}$ and $B: Y \rightarrow 2^{X}$ by

$$
A(x)=\left\{y \in Y: f(x, y)>\lambda_{\varepsilon}\right\} \quad \text { and } \quad B(y)=\left\{x \in X: g(x, y)<\lambda_{\varepsilon}\right\}
$$

for each $x \in X$ and $y \in Y$, respectively. Note that both $A$ and $B$ have (maybe empty) convex values and open fibers and, moreover, they do not have any coincident point. Otherwise there exists a pair $\left(x_{0}, y_{0}\right) \in X \times Y$ such that $\lambda_{\varepsilon}<f\left(x_{0}, y_{0}\right) \leq g\left(x_{0}, y_{0}\right)<\lambda_{\varepsilon}$, 
which is impossible. By Theorem 3.1, we must have that either $A$ or $B$ is not a KF mapping. As $A(x) \neq \emptyset$ for each $x \in X$, there must exist an element $y_{0} \in Y$ such that $B\left(y_{0}\right)=0$. Therefore $\inf _{x \in X} g\left(x, y_{0}\right) \geq \lambda_{0}-\varepsilon$, so that $\sup _{y \in Y} \inf _{x \in X} g(x, y) \geq \lambda_{0}-\varepsilon$. As $\varepsilon$ is arbitrary, we have $\sup _{y \in Y} \inf _{x \in X} g(x, y) \geq \lambda_{0}$. Then

$$
\sup _{y \in Y} \inf _{x \in X} g(x, y) \geq \min _{x \in X} \sup _{y \in Y} f(x, y)
$$

and the proof is complete

As applications of Fan's lemma in [9] which is an infinite dimensional extension of the classical Knaster-Kuratowski-Mazurkiewicz lemma in [13], a number of minimax inequalities have been given by Aubin [1], Fan [9 - 10], Lassonde [14], Park [18], Sion [20], Tan and Yuan [21] and others. For more details, the interested readers are referred to Simons' paper [19]. The coincidence theorems known in algebraic topology consequently give rise to corresponding minimax theorems (see Simons [19] and references cited there). There are also a number of literatures for the study of coincidence theorems (see, e.g., Dugundji and Granas [8], Granas and Liu [11], Park [18], and Tarafdar and Yuan [23] in this direction. Finally, we would like to remark that it is possible to give a family version of Ky Fan type minimax inequalities (see, e.g., Fan [10]), and Hartman-Stampacchia and Browder type variational inequalities in product spaces. In fact, some results in this line have been established in the joint research work [6] and thus we omit these details here.

Acknowledgment. Both authors would like to thank anonymous referees' careful reading and critical suggestion which lead to the present version of this paper, and in particular to lead us to give a correct version of the proof for Theorem 3.2.

\section{References}

[1] Aubin, J. P.: Mathematical Methods of Game and Economic Theory. Rev. edit. Amsterdam: North - Holland 1982.

[2] Ben-El-Mechaiekh, H. and P. Deguire: Maximal elements for families of multifunctions. Moncton (Canada): University Res. Rep. MATH-27 (1994), 1- 17.

[3] Browder, F. E.: The fixed point theory of multivalued mappings in topological vector spaces. Math. Ann. 177 (1968), 283 - 301.

[4] Deguire, P.: Browder-Fan fixed point theorem and related results. Research report. Moncton (Canada): University Res. Rep. MATH-26 (1993), $1-16$.

[5] Deguire, P. and M. Lassonde: Sélections et points fixes généralisés dans des espaces produits. Moncton (Canada): University Res. Rep. MATH-25 (1993), 1 - 27.

[6] Deguire, P., Tan, K. K. and X. Z. Yuan: Some maximal elements, fixed points and equilibria for $L_{S}$-majorizd mappings in product spaces and its applications. Research Report. Dalhousie University (Canada) MATH-August 1 (1993), 1 - 32.

[7] Ding, X. P., Kim, W. and K. K. Tan: Equilibria of non-compact generalized games with $L^{*}$-majorized preference correspondences. J. Math. Anal. Appl. 164 (1992), 508 - 517.

[8] Dugundji, J. and A. Granas: Fixed Point Theory, Vol. I. Warsaw: Polish Sci. Publ. 1982. 
[9] Fan, K.: A generalization of Tychonoff's fixed point theorem. Math. Ann. 142 (1961), $305-310$.

[10] Fan, K.: A minimax inequality and applications. In: Inequalities. Proc. 3d Symp. Inequ. Los Angeles, California Univ., Sept. 1 - 9, 1969 (ed.: O. Shisha). New York: Academic Press 1972, pp. $103-113$.

[11] Granas, A. and F. C. Liu: Coincidences or set-valued maps and minimax inequalities. J. Math. Pure. Appl. 65 (1986), 119 - 148.

[12] Hildenbrand, W. and H. Sonnenschein: Handbook of Mathematical Economics, Vol. 4. Amsterdam: North-Holland 1993.

[13] Knaster, B., Kuratowski, C. and S. Mazurkiewicz: Ein Beweis des Fixpunktsatzes für n-dimensionale Simplexe. Fund. Math. 14 (1929), 132 - 137.

[14] Lassonde, M.: On the use of $K K M$ multifunctions in fixed point theory and related topics. J. Math. Anal. Appl. 97 (1983), 151 - 201.

[15] Lassonde, M. and C. Schenkel: KKM principle, fixed points and Nash equilibria. J. Math. Anal. Appl. 164 (1992), 542 - 548.

[16] Mas-Colell, A.: An equilibrium existence theorem without complete or transitive preferences. J. Math. Economics 1 (1974), 237 - 346.

[17] Mas-Colell, A.: The Theory of General Economic Equilibria: a Differential Approach. Cambridge: Univ. Press 1985.

[18] Park, S.: Some coincidence theorems on acyclic multifunctions and applications to KKM theory. In: Fixed Point Theory and Applications (ed.: K.-K. Tan). River Edge (N.J., USA): World Sci. 1992, pp. 248 - 277.

[19] Simons, S.: Minimax theorems and their proofs. Preprint. Santa Barbara (USA): Univ. California 1994.

[20] Sion, M.: On general minimax theorems. Pac. J. Math. 8 (1958), $171 \cdot 176$.

[21] Tan, K. K. and X. Z. Yuan: A minimax inequality with applications to existence of equilibrium points. Bull. Austral. Math. Soc. 47 (1993), 483 - 503.

[22] Tarafdar, E.: A fixed point theorem and equilibrium points of an abstract economy. J. Math. Economics 20 (1991), 211 - 218.

[23] Tarafdar, E. and X. Z. Yuan: A remark on coincidence theorems. Proc. Amer. Math. Soc. 122 (1994), $957-960$.

[24] Toussaint, S.: On the existence of equilibria in economies with infinitely many commodities and without ordered preferences. J. Econ. Theory 33 (1984), 98 - 115.

[25] Tulcea, C. I.: On the approximation of upper-semicontinuous correspondences and the equilibriums of generalized games. J. Math. Anal. Appl. 136 (1988), 267 - 289.

[26] Yannelis, N. C. and N. D. Prabhakar: Existence of maximal elements and equilibria in linear topological spaces. J. Math. Economics 12 (1983), $233-245$.

Received 28.12.1994; in revised form 25.07.1995 